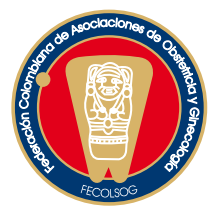

Reporte de caso

\title{
ARTROGRIPOSIS MÚLTIPLE CONGÉNITA EN GEMELO MONOCORIÓNICO BIAMNIÓTICO. REPORTE DE CASO Y REVISIÓN DE LA LITERATURA
}

\section{A case report of arthrogryposis multiplex congenita in monochorionic biamniotic twins}

\author{
Felipe Ruiz-Botero*, Harry Pachajoa M.D., Ph.D(A)**, \\ Wilmar Saldarriaga M.D., M.Sc***, Carolina Isaza M.D., M.Sc****
}

Recibido: julio 30/08 - Aceptado: enero 30/09

\section{RESUMEN}

Introducción: la artrogriposis múltiple congénita es un grupo de desórdenes musculoesqueléticos de muy rara aparición, caracterizados por contracturas múltiples articulares, en los que se afectan los músculos de los miembros superiores, inferiores y del dorso. La etiología de esta condición permanece desconocida y muchos agentes causales han sido implicados, particularmente infecciones virales y condiciones restrictivas intrauterinas.

Objetivo: aportar a la literatura un caso de artrogriposis múltiple congénita (AMC) en un embarazo gemelar monocoriónico biamniótico, y revisar la etiología y fisiopatogenia de esta malformación congénita.

Presentación del caso: en el segundo gemelo de sexo masculino, con un peso de 1345 g, se observó acortamiento de las cuatro extremidades de todos

* Estudiante de tercer año, Escuela de Medicina, Facultad de Salud, Universidad del Valle. Cali, Colombia.

** Profesor Auxiliar, Aspirante a Doctor en Ciencias Biomédicas, Facultad de Salud, Universidad del Valle. Grupo de Malformaciones Congénitas Perinatales y Dismorfología HUV. Cali, Colombia. Correo electrónico: harrympl@yahoo.com

*** Profesor asistente, Escuela de Ciencias Básicas Médicas, Universidad del Valle, Ginecólogo y Obstetra, M.Sc. en Embriología y Genética. Fundación Valle del Lili. Hospital Universitario del Valle. Cali, Colombia.

**** Profesora Titular, Departamento de Morfología, Facultad de Salud, Universidad del Valle. Grupo de Malformaciones Congénitas Perinatales y Dismorfología HUV. Cali, Colombia. los segmentos, contracturas articulares que llevaban a una desviación marcada de manos y pies hacia la línea media, e imposibilidad de los movimientos activos.

Discusión: el presente reporte describe AMC en uno de los gemelos idénticos, lo cual sugiere que esta condición no se presentó por alteraciones cromosómicas o enfermedades hereditarias, sino por uno o varios factores extrínsecos al feto, probablemente de origen mecánico, que pueden comprometer el desarrollo normal de las articulaciones y llevar a la aparición de AMC. En la literatura revisada, éste es el cuarto reporte de características similares.

Palabras clave: artrogriposis, enfermedades en gemelos, defectos congénitos.

\section{SUMMARY}

Arthrogryposis multiplex congenita (AMC) is a group of rarely occurring musculoskeletal disorders, characterised by multiple joint contractures affecting the muscles of the upper and lower limbs and the back. This condition's aetiology remains unknown and many causal agents have been implicated, particularly viral infections and intra-uterine restrictive conditions.

Objective: contributing a case of AMC in a monochorionic biamniotic twin pregnancy to the literature 
and reviewing this congenital malformation's aetiology and physiopathogeny.

Case presentation: a second-born male twin, weighing $1345 \mathrm{gr}$, shortening of the four limbs in all segments was observed, accompanied by joint contractures leading to marked hand and foot deviation towards the mid line and the impossibility of active movement.

Discussion: AMC being displayed in one of the identical twins mentioned in the present report suggests that this condition did not take place because of chromosomal alteration or a hereditary disease. Rather, it was presented because of one or several external factors (probably mechanical) affecting the foetus which may have jeopardised the normal development of the joints and led to AMC appearing. This is the fourth report of similar characteristics in the literature reviewed.

Key words: arthrogryposis, diseases in twins, birth defect.

\section{INTRODUCCIÓN}

La artrogriposis múltiple congénita es un grupo de desórdenes musculoesqueléticos de muy rara aparición, caracterizados por contracturas múltiples articulares, en los que se afectan los músculos de los miembros superiores, inferiores y del dorso. Los músculos pueden estar ausentes, reducidos en tamaño y/o número, o ser reemplazados por tejido fibroso o grasa. ${ }^{1}$ El grado de afectación varía de paciente a paciente y la deformidad clásica es bilateral y simétrica. $^{2}$

La prevalencia de la AMC, en su forma leve, se ha estimado en 3 de cada 1000 recién nacidos, pero su forma clásica afecta a $0,03 \%$ de los neonatos. No existe diferencia asociada a raza o sexo y sólo en 30\% de los casos se encuentra una causa genética. ${ }^{3}$

La etiología de esta condición permanece desconocida. ${ }^{4}$ En la literatura revisada se reportaron 3 casos de artrogriposis múltiple congénita, producto de embarazos gemelares monocigóticos. ${ }^{2,5}$ Nosotros queremos aportar un caso de AMC en un embarazo gemelar monocoriónico biamniótico, y revisar la etiología y fisiopatogenia de esta malformación, las cuales son de importancia conceptual para los obstetras que hacen ultrasonografía y para quienes interpretan los resultados en consulta, lo que permitirá un mejor abordaje con los pacientes.

\section{METODOLOGÍA}

Basados en el interrogante de la presentación de casos con AMC en embarazos gemelares monocoriónicos biamnióticos, se realizó una búsqueda de la literatura en Internet, en las bases de datos de PubMed/Medline, BIREME y LILACS, de los años 1995 a 2008, con las palabras clave: arthrogryposis, diseases in twins, birth defect.

\section{REPORTE DE CASO}

Recién nacido masculino, producto de embarazo de padre de 26 años y madre de 14 años, con 2 embarazos previos, un parto y un aborto. La madre acude a nuestra institución por inicio de trabajo de parto a las 36 semanas de edad gestacional y embarazo gemelar monocoriónico biamniótico por ecografía obstétrica del segundo y tercer trimestre, que no evidenció anormalidades. Sin antecedentes de importancia en el período periconcepcional, ni en el control prenatal. Se realizó cesárea a las 36 semanas por trabajo de parto pretérmino y presentación distócica; el primer gemelo masculino pesó 1150 g y no presentó anormalidades externas. El segundo gemelo de sexo masculino pesó 1345 g, presentó hirsutismo facial, en extremidades y dorso; además, filtrum largo, micrognatia, diástasis de recto abdominal, criptorquidia bilateral, pene generoso, líneas palmares ausentes y hoyuelos en dorso de muñecas (figura 1). Además, se observó acortamiento de las cuatro extremidades de todos los segmentos, contracturas articulares que llevaban a una desviación marcada de manos y pies hacia la línea media, e imposibilidad de los movimientos activos en las articulaciones (figuras 1 y 2). 
Figura 1. Vista anterior de recién nacido producto de embarazo gemelar monocoriónico biamniótico con artrogriposis múltiple congénita. Nótese la artrogriposis de las articulaciones de las extremidades, la diástasis de rectos abdominales y el pene generoso.

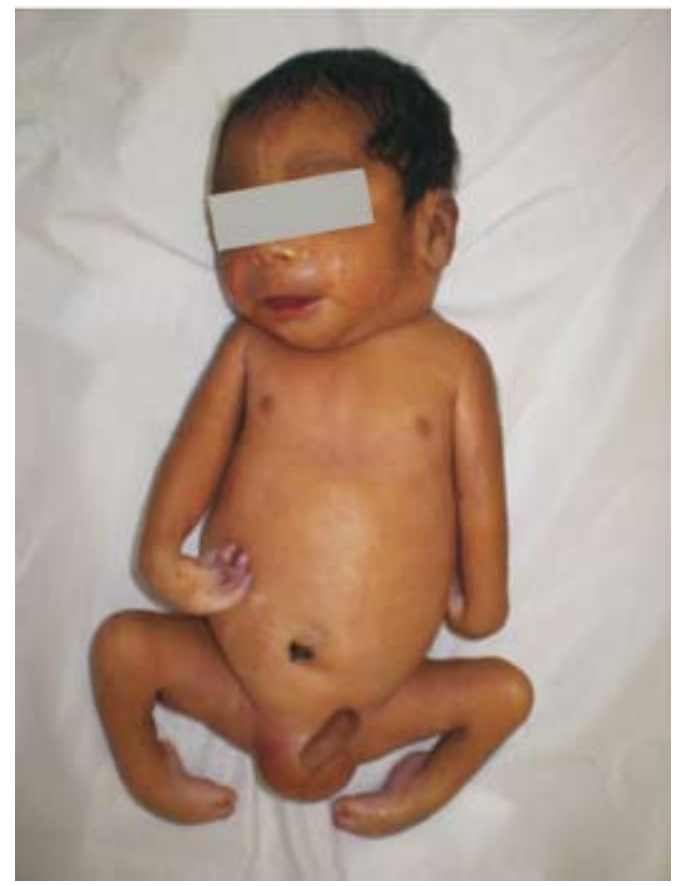

Figura 2. Vista lateral de recién nacido producto de embarazo gemelar monocoriónico biamniótico con artrogriposis múltiple congénita. Nótese la ausencia de líneas producidas por la articulación del codo y el hirsutismo en las extremidades.

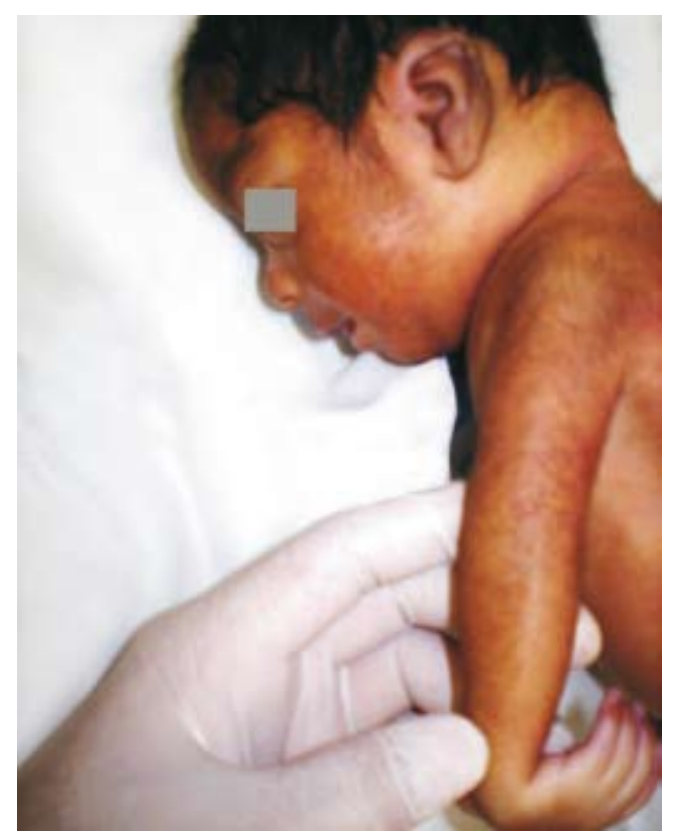

\section{DISCUSIÓN}

En la literatura revisada se encontraron tres reportes de AMC en embarazos múltiples. Sin embargo, el presente reporte describe AMC en uno de los gemelos idénticos, lo cual sugiere que esta condición no se presentó por alteraciones cromosómicas o enfermedades hereditarias, sino por uno o varios factores extrínsecos al feto (ver adelante mecanismo fisiopatológico), que pueden comprometer el desarrollo normal de las articulaciones y llevar a la aparición de AMC.

\section{Etiología}

Se ha informado que la artrogriposis múltiple congénita hace parte de un espectro de características que pueden presentarse como hallazgo único o ser parte de un síndrome específico, existen aproximadamente 150 síndromes de origen genético. ${ }^{6}$

La posible etiología de esta condición permanece desconocida, pero una teoría plantea que este defecto congénito es producido por un daño desigual de las células del cuerno o asta anterior de la médula espinal durante el desarrollo fetal. Esto explicaría las alteraciones en las extremidades en los recién nacidos afectados. La causa de este evento disruptivo durante la vida embrionaria y fetal no está clara, pero se han postulado hipótesis como infecciones por virus del herpes simplex, insuficiencia placentaria, reacciones de estrés y alteraciones en la regulación térmica del feto. ${ }^{7}$

\section{Mecanismo fisiopatológico}

Durante el desarrollo embrionario de las articulaciones, que se inicia en la semana 5 y 6 de gestación, es necesaria una adecuada movilidad de las articulaciones para el desarrollo de éstas y de estructuras aledañas, como el tejido conectivo y los tendones que están en formación. ${ }^{8}$ En los casos donde la movilidad sea mínima o nula, por factores intrínsecos o extrínsecos, se puede producir una alteración que puede llevar a la artrogriposis. ${ }^{9}$ 
Los factores intrínsecos que están asociados a la AMC incluyen enfermedades del sistema nervioso central, como las malformaciones cerebrales. ${ }^{10}$

Entre los factores extrínsecos se encuentran los relacionados con alteraciones de la morfología de la cavidad uterina, una cavidad pequeña. Una malformación uterina puede generar poco espacio en la cavidad uterina, lo cual lleva a una mínima movilidad de las articulaciones del feto.,

En los embarazos múltiples, la cavidad uterina debe ser compartida para cada uno de los fetos en desarrollo, disminuyendo así el espacio que permite una adecuada movilidad de las articulaciones, llevando así a la ocurrencia de AMC.

En nuestro caso, por ser producto de un embarazo gemelar monocoriónico biamniótico de una madre de 14 años, estas dos condiciones podrían llevar a una disminución significativa del espacio disponible para realizar movimientos fetales y el desarrollo adecuado del sistema osteomuscular.

\section{REFERENCIAS}

1. Alves PV, Zhao L, Patel PK, Bolognese AM. Arthrogryposis: diagnosis and therapeutic planning for patients seeking orthodontic treatment or orthognathic surgery. J Craniofac Surg 2007;18:838-43.
2. Mennen U. Arthrogryposis multiplex congenita: functional classification and the AMC disc-o-gram. J Hand Surg 2004;29:363-7.

3. López R, Gutiérrez M, Quero O, Méndez M. Artrogriposis múltiple congénita. Reporte de un caso. MedULA 2003;9:61-4.

4. Mennen U, Williams E. Arthrogryposis multiplex congenita in a monozygotic twin an intrauterine lesion? J Hand Surg 1996;21:647-8.

5. Hillman JW, Jonson TH. Arthrogryposis multiplex congenita in twins. J Bone Joint Surg Am 1952;34:211-4.

6. Bürglen L, Amiel J, Viollet L, Lefebvre S, Burlet P, Clermont, et al. Survival motor neuron gene deletion in the arthrogryposis multiplex congenitaspinal muscular atrophy association. J Clin Invest 1996;98:1130-2.

7. Mennen U, van Heest A, Ezaki MB, Tonkin M, Gericke G. Arthrogryposis multiplex congenita. J Hand Surg 2005;30:468-74.

8. Drachman DB, Banker BQ. Arthrogryposis multiplex congenita. Case due to disease of the anterior horn cells. Arch Neurol 1961;5:77-93.

9. Drachman DB, Coulombre AJ. Experimental clubfoot and arthrogryposis multiplex congenita. Lancet 1962;15:523-6.

10. Saito Y, Hayashi M, Miyazono Y, Shimogama T, Ohno K. Arthrogryposis multiplex congenita with callosal agenesis and dentato-olivary dysplasia. Brain Dev 2006;28:261-4. 